\title{
Impact of hydrogenation conditions on the temperature and strain coefficients of type 1 and type 1a dual grating sensors
}

K. Kalli, George Simpson, Kaiming Zhou, Lin Zhang, Ian Bennion

K. Kalli, George Simpson, Kaiming Zhou, Lin Zhang, lan Bennion, "Impact of hydrogenation conditions on the temperature and strain coefficients of type 1 and type 1a dual grating sensors," Proc. SPIE 5855, 17th International Conference on Optical Fibre Sensors, (23 May 2005); doi: $10.1117 / 12.624287$

SPIE Event: 17th International Conference on Optical Fibre Sensors, 2005, Bruges, Belgium 


\title{
Impact of hydrogenation conditions on the temperature and strain coefficients of type 1 and type1a dual grating sensors
}

\author{
Kyriacos Kalli*a ${ }^{\mathrm{a}}$, George Simpson ${ }^{\mathrm{b}}$, Kaiming Zhou ${ }^{\mathrm{b}}$, Lin Zhang ${ }^{\mathrm{b}}$, Ian Bennion ${ }^{\mathrm{b}}$ \\ ${ }^{a}$ Higher Technical Institute, C. Kavafi Street, Nicosia, 2152 Cyprus \\ ${ }^{\mathrm{b}}$ Photonics Research Group, Aston University, Birmingham B4 7ET United Kingdom
}

\begin{abstract}
We report experimental findings for tailoring the temperature and strain coefficients of Type 1 and Type 1A fibre Bragg gratings by influencing the photosensitivity presensitisation of the host optical fibre. It is shown that by controlling the level of hydrogen saturation, via hot and cold hydrogenation, it is possible to produce gratings with lower thermal coefficients. Furthermore, there is a larger difference between the Type 1 and Type 1A thermal coefficients and a significant improvement in the matrix condition number, which impacts the ability to recover accurate temperature and strain invariant data using the Type1-1A dual grating sensor.
\end{abstract}

Keywords: Optical fibre sensors, fibre Bragg gratings, type 1A grating, photosensitivity, temperature and strain sensors

\section{INTRODUCTION}

Type IA fibre Bragg gratings have attracted interest within the sensor community based on their unique spectral and physical characteristics. They are readily identified by their spectral attribute of a large red shift in the Bragg wavelength $(\mathrm{B})$ of the grating during inscription that accompanies an increase in the mean core index ${ }^{1}$. It is recognised that this red shift is dependent on fibre type and hydrogenation conditions, and for a highly doped fibre, such as B/Ge codoped fibre, is typically $15-20 \mathrm{~nm}$, whereas the wavelength shift for SMF28 is lower at $5-8 \mathrm{~nm}^{2}$. The maximum wavelength shift translates to an increase in the mean index of up to $2 \times 10^{-2}$. We have previously shown a strong correlation between the growth of the $\mathrm{OH}$ absorption band formation in the optical fibre during prolonged UV exposure and the increase in the mean index change of the fibre grating. This change results from the hydrogen combining with $\mathrm{Si}$ and Ge centres in the fibre to form stable $\mathrm{SiOH}$ and $\mathrm{GeOH}$ groups, the latter of which has the greater impact on the strength and peak location of the 1400nm absorption band. As a result of this fundamental material modification Type 1 and Type 1A gratings have been written in the same fibre with a common phase mask, yet with central reflecting peaks more than 14nm apart after annealing. More importantly, this change in the mean index of the fibre core results in their key physical attribute, that they exhibit the lowest temperature coefficient of all grating types reported to date; this makes them attractive for use in a temperature compensating, dual grating sensor ${ }^{3}$. Here we highlight the effect of hydrogenation on Type 1 and Type $1 \mathrm{~A}$ grating thermal coefficients and how this affects the recovery of accurate temperature and strain data.

\section{HOT OR COLD HYDROGENATION?}

In order to determine if the type of hydrogenation has an effect on the formation of the Type 1 and Type IA gratings, two identical batches of fibre were prepared. Each batch consisted of Corning SMF28 standard telecommunications fibre and Verillion B/Ge co-doped fibre. One batch was hydrogenated at $80^{\circ} \mathrm{C}, 190 \mathrm{Bar}$ for 93 hours and cooled to room temperature over 24 hours by which time the pressure was 160Bar; the other batch was hydrogenated in excess of four months at 180Bar and at room temperature. Optical fibres hydrogenated at lower temperatures achieve a higher hydrogen concentration within the core but require significantly longer saturation times ${ }^{4}$. Equation 1 is used to calculate the saturation level of hydrogen molecules within the core,

$$
\kappa_{\text {sat }}=3.3481 \mathrm{pexp}\left[\frac{8670 \mathrm{~J} / \mathrm{mol}}{R T}\right]
$$

*kkalli@hti.ac.cy; phone +35722406537; fax +35722406545; www.hti.ac.cy 
where $\kappa_{\text {sat }}$ is the equilibrium solubility of the hydrogen, $p$ is the pressure of the hydrogen in atmospheres, $T$ is the temperature in Kelvin, and $R$ is the gas constant $(8.31451 \mathrm{~J} / \mathrm{K} / \mathrm{mol})$. Both samples were hydrogenated for times well in excess of the equilibrium time and we calculated the hydrogen concentration within the fibre samples using equation 1 based on the hydrogenation conditions outlined above. These results are shown in Table 1.

\begin{tabular}{c|ccc|c}
\hline & Temperature (K) & Pressure (Atmosphere) & Time (Hours) & $\kappa_{\text {sat }}(\mathrm{ppm})$ \\
\hline Hot & 353 & 178 & 93 & 11400 \\
Cold & 298 & 188 & $>384$ & 21000 \\
\hline
\end{tabular}

Table 1. A summary of the hydrogenation conditions for the hot and cold hydrogenated samples showing the time, temperature and pressure of hydrogenation and the calculated saturation level within the fibre core.

Gratings were inscribed using the scanning phase mask technique illuminated with a cw UV laser source operating at $244 \mathrm{~nm}$. A $1 \mathrm{~mm}$ aperture ensured an accurate top-hat exposure profile along the length of the grating ${ }^{3}$. The stage was scanned at $1 \mathrm{mms}^{-1}$ with $130 \mathrm{~mW}$ optical power delivered to the fibre. Figure 1 shows the growth rate for the hot and cold samples in the different fibre types. There is a clear trend showing that the fibres hydrogenated at room temperature grow faster then those heated during the hydrogenation process.
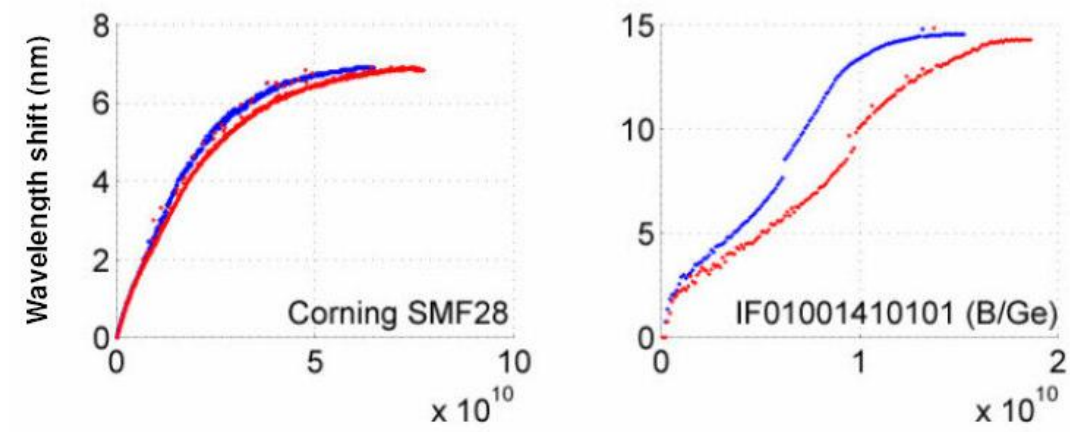

Fluence $\left(\mathrm{J} / \mathrm{m}^{2}\right)$

Figure 1. Growth rate of Type IA gratings in the indicated fibre types, for hot (grey) and cold (black) hydrogenation conditions.

In order that the IA gratings have a reference grating whose properties are well understood, a 1 mm Type I grating was written within the same section of each fibre. Figure 2 shows the spectra of each sample before (upper) and after (lower) annealing at $80^{\circ} \mathrm{C}$ for 96 hours, and highlights a number of differences between the fabricated gratings; notable examples are the gratings written in Verillion IF01001410101 B/Ge co-doped fibre, where both the IA and I gratings differ significantly in amplitude and the Type I grating would seem to be slightly offset in wavelength. The gratings in SMF28 are comparable in hot and cold hydrogenated samples and do not exhibit any significant spectral characteristics. The wavelength difference between Bragg resonances of the Type I and IA gratings is summarised in Table 2 for the pre- and post-annealed gratings.

\section{TEMPERATURE AND STRAIN COEFFICIENTS - CONDITION NUMBER}

The thermal and strain coefficients of the gratings were measured by placing individual gratings on a temperature controlled block within an insulated chamber and mounted on translation stages. The temperature was controlled by means of a Peltier device connected to a standard, computer operated temperature controller. Temperature feedback was made possible by the placement of a calibrated thermistor and the Bragg wavelength was measured by passing broadband IR radiation from a powerful ASE source through the fibre to an OSA with 0.06nm resolution. The centroidfitting algorithm (CFA) was used to locate the Bragg wavelength peak recorded by the OSA. A computer was used to set and record the temperature of the grating and the OSA traces; plotted in Figure 3 for each grating and fibre type, as indicated. The thermal coefficients are summarised in Table 2. 

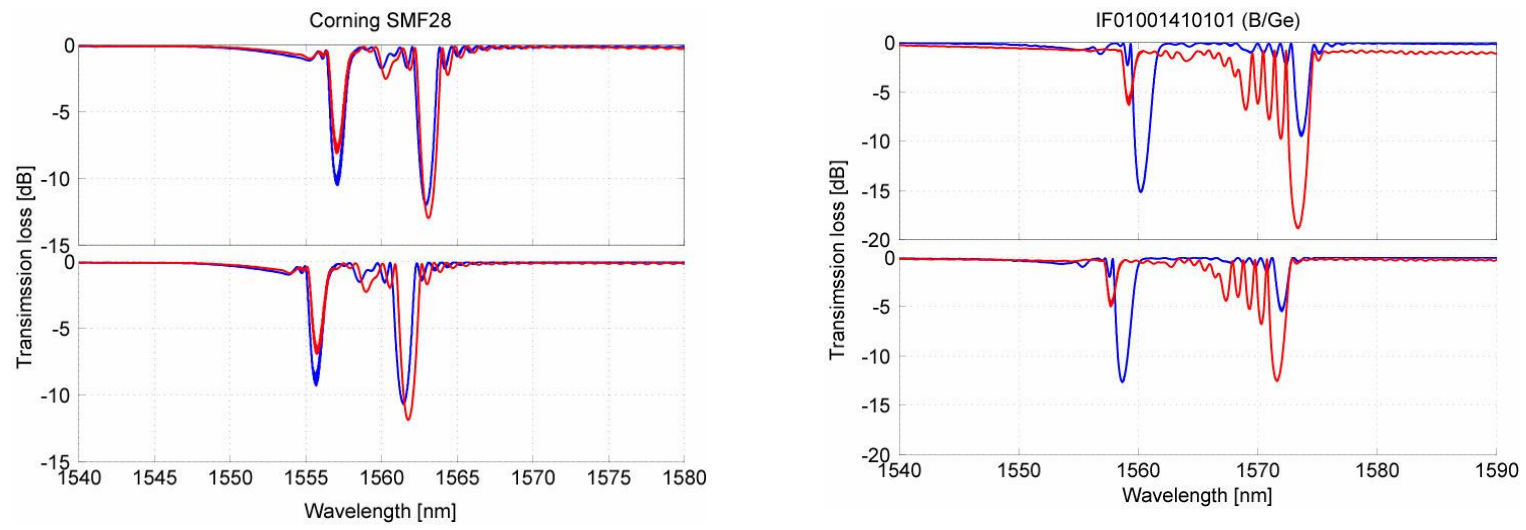

Figure 2. Pre- (upper traces) and post- (lower traces) annealing spectra for Type I and Type IA gratings written in various fibre types (as indicated) for hot (grey) and cold (black) hydrogenation conditions.

\begin{tabular}{|c|c|c|c|c|c|c|c|}
\hline Fibre type & $\begin{array}{l}\text { Hydrogenation } \\
\text { conditions }\end{array}$ & Manufacturer & $\begin{array}{l}\lambda_{B R}^{I}(T) \\
\mathrm{pm}^{\circ} \mathrm{C}^{-1}\end{array}$ & $\begin{array}{l}\lambda_{B R}^{I A}(T) \\
\mathrm{pm}^{\circ} \mathrm{C}^{-1}\end{array}$ & $\begin{array}{c}\frac{\lambda_{B R}^{I}(T)}{\lambda_{B R}^{I A}(T)} \\
\%\end{array}$ & $\begin{array}{c}\lambda_{B R}^{I A}-\lambda_{B R}^{I} \\
\mathrm{~nm}\end{array}$ & $\begin{array}{c}\lambda_{B R}^{I A}-\lambda_{B R}^{I} \\
\text { annealed } \\
\mathrm{nm}\end{array}$ \\
\hline $\begin{array}{l}\text { SMF28 } \\
\text { SMF28 }\end{array}$ & $\begin{array}{l}\text { hot } \\
\text { cold }\end{array}$ & $\begin{array}{l}\text { Corning } \\
\text { Corning }\end{array}$ & $\begin{array}{l}10.54 \\
10.28\end{array}$ & $\begin{array}{c}10.427 \\
9.958\end{array}$ & $\begin{array}{l}1.084 \\
3.233\end{array}$ & $\begin{array}{l}5.848 \\
6.076\end{array}$ & $\begin{array}{l}5.761 \\
6.032\end{array}$ \\
\hline $\mathrm{B} / \mathrm{Ge}$ & hot & $\begin{array}{l}\text { Verillion } \\
\text { Verillion }\end{array}$ & $\begin{array}{l}9.146 \\
8531\end{array}$ & $\begin{array}{c}8.27 \\
7.403\end{array}$ & $\begin{array}{l}10.592 \\
15.237\end{array}$ & $\begin{array}{l}14.147 \\
13474\end{array}$ & $\begin{array}{l}13.925 \\
13.375\end{array}$ \\
\hline
\end{tabular}

Table2. A summary of the data highlighting the differences between hot and cold hydrogenation in the different fibre types.

Figure 3 shows that both the hot and cold hydrogenated fibres display an unambiguous trend, the cold samples possess a lower temperature coefficient $\left({ }_{B R}(T)\right)$. Moreover the difference between the ${ }_{B R}(T)$ values for Type I and IA gratings is larger when the samples are hydrogenated at $25^{\circ} \mathrm{C}$ in preference to $80^{\circ} \mathrm{C}$. It is unclear from this experiment alone whether the effect is a result of an increased hydrogen level caused by the longer term, lower temperature hydrogenation. However, it is almost certainly related to the presence of increased $\mathrm{GeOH}$ centres that are formed during the UV treatment of the fibre. The wavelength to strain responsivity displays a smaller but measurable difference that results primarily from differences in the initial centre wavelengths rather than any variation in the material properties. For the case of the Verillion B/Ge fibre the strain coefficients for the Type 1 and Type $1 \mathrm{~A}$ gratings are ${ }^{I}{ }_{B R}()^{\prime}=0.818 \mathrm{pm} / \quad$ and

${ }_{B R}()=0.828 \mathrm{pm} /$, respectively. The strain and temperature errors associated with their respective coefficients are $\pm 15.3 / \mathrm{pm}$ and $\pm 1.44^{\circ} \mathrm{C} / \mathrm{pm}$, for the hot hydrogenation in Verillion $\mathrm{B} / \mathrm{Ge}$ fibre and $\pm 11.2 \mathrm{Jm}$ and $\pm 1.15^{\circ} \mathrm{C} / \mathrm{pm}$ for the cold hydrogen loading in the same fibre type. These values compare with errors of $\pm 12 / \mathrm{pm}$ and $\pm 1.3^{\circ} \mathrm{C} / \mathrm{pm}$ measured by $\mathrm{Xu}$ et al. ${ }^{5}$ for two superimposed gratings at markedly different wavelengths. Therefore the approach of controlling the degree of hydrogenation to affect the temperature coefficients of a photosensitive fibre can prove to be very useful for a dual grating sensor used to differentiate between strain and temperature. Furthermore, a useful mathematical tool for quickly assessing the accuracy of a dual sensor arrangement is to calculate the condition number of the sensor matrix ${ }^{6}$. The condition number of a matrix measures the sensitivity of the solution to errors in the data. For the ideal case the condition number of the matrix is 1 indicating a perfectly conditioned matrix. Table 3 shows a comparison of condition numbers for different methods used to separate temperature and strain based on Bragg grating sensors, from which we observe that the cold hydrogenation produces a well-conditioned matrix that compares favourably with other more elaborate techniques.

\section{CONCLUSIONS}

We have demonstrated that controlling the level of hydrogen used to increase the photosensitivity of an optical fibre can control the temperature to wavelength responsivity of the grating sensor. In the case of low temperature hydrogenation there is a significant improvement in the matrix inversion errors and the condition number. This improvement is 
important as it augments the existing advantages of the Type1-1A dual grating sensor, namely two Bragg wavelengths having good wavelength proximity thereby avoiding costly multiplexing schemes; quick and efficient inscription using a single phase mask, common annealing cycles and the precise placement of sensors located in a common sensor head.
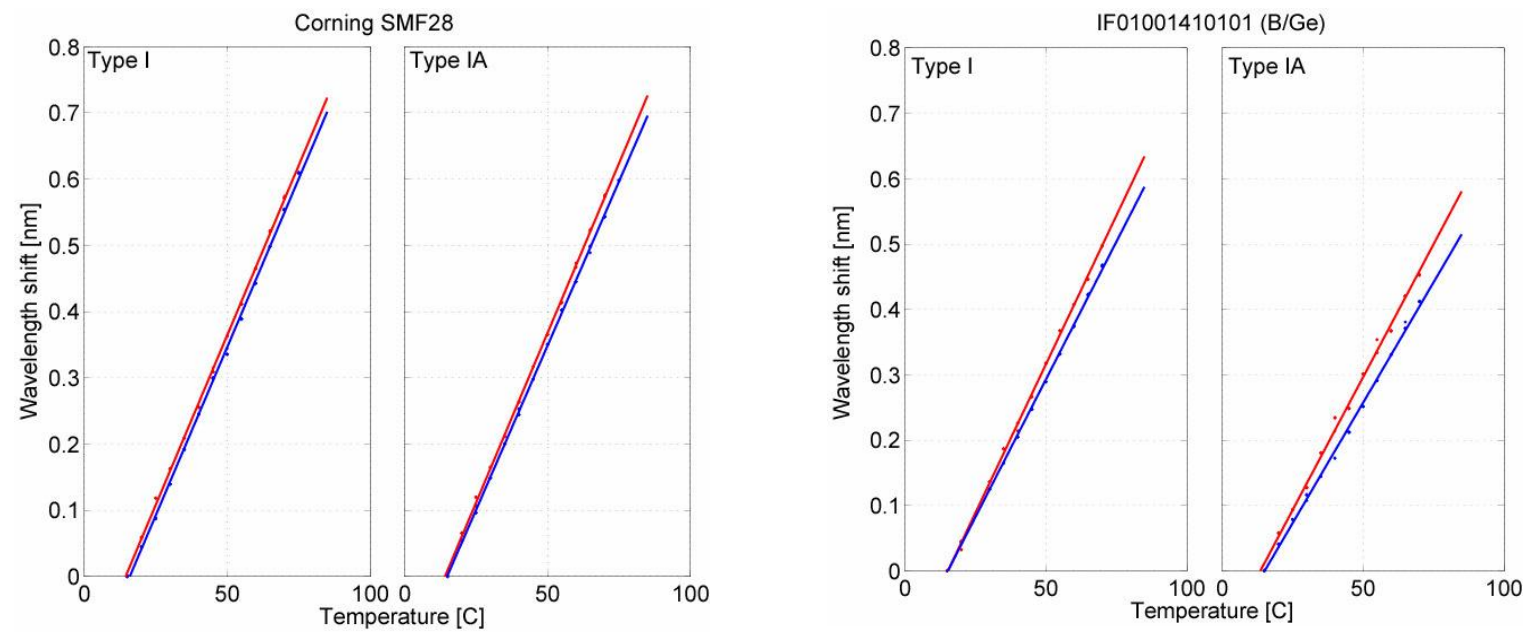

Figure 3. Plots showing the thermal coefficients for Type I (left-hand traces) and Type IA (right-hand traces) gratings written in various fibre types (as indicated) for hot (grey) and cold (black) hydrogenation conditions.

\begin{tabular}{c|c|c}
\hline Method & Reference 6 and references therein & Condition number \\
\hline Type1-1A grating pair - Cold / Hot hydrogenation & This work & $147 / 214$ \\
Bragg gratings in different diameter fibres & James et al & 48 \\
Type IIA and Type IA grating pair & Shu et al & 68 \\
Superimposed grating pair & Xu et al & 123 \\
First and second order Bragg resonances & Sivanesan et al / Kalli et al & $161 / 203$ \\
Gratings in germanosilicate and B doped germanosilicate & Cavalerio et al & 173 \\
\hline
\end{tabular}

Table 3. Matrix condition numbers of dual grating temperature-strain isolation configurations - a comparison.

\section{ACKNOWLEDGEMENTS}

The authors acknowledge the UK DTI-EPSRC LINK project EMPIRE. KK and AGS respectively acknowledge Higher Technical Institute - Promotion of Research Fund and studentship of the UK EPSRC and BAE Systems.

\section{REFERENCES}

1. A.G. Simpson, K. Kalli, K. Zhou, L. Zhang and I. Bennion, "Formation of type IA fibre Bragg gratings in germanosilicate optical fibre," Electronics Letters, Vol. 40, p 163-164, 2004.

2. A.G. Simpson, K. Kalli, L. Zhang, K. Zhou and I. Bennion, "Abnormal photosensitivity effects and the formation of type IA FBGs,” BGPP, Monterey, California, MD31, 2003.

3. A.G. Simpson, K. Kalli, K. Zhou, L. Zhang and I. Bennion, "An idealised method for the fabrication of temperature invariant IA-I strain sensors," postdeadline session, OFS16 Nara, Japan, PD4, 2003.

4. P. Lemaire, "Reliability of optical fibres exposed to hydrogen: prediction of long-term loss increases," Optical Engineering, Vol. 30, p 780-781, 1991.

5. M. G. Xu, J. L. Archambault, L. Reekie, and J. P. Dakin, "Discrimination between strain and temperature effects using dual wavelength fibre sensors," Electronics Letters, Vol. 30, p 1085-1087, 1994.

6. P. Sivanesan, JS. Sirkis, Y. Murata, and S. Buckley, "Optimal wavelength pair selection and accuracy analysis of dual fibre grating sensors for simultaneously measuring strain and temperature," Optics Engineering, Vol. 41, p 2456-2463, 2002, and references therein. 